Pure and Applied Mathematics Quarterly

Volume 7, Number 2

(Special Issue: In honor of

Frederick W. Gehring, Part 2 of 2$)$

345 - 363,2011

\title{
New Results for p Harmonic Functions
}

\author{
John L. Lewis* and Kaj Nyström \\ Dedicated to Fred Gehring on his eighty first birthday.
}

\begin{abstract}
In this paper we first discuss new results of the authors concerning a boundary Harnack inequality and Hölder continuity up to the boundary for the ratio of two positive $p$ harmonic functions, $1<p<\infty$, which vanish on a portion of a Lipschitz domain. Second we discuss applications of these results to the Martin boundary problem for $p$ harmonic functions and to certain boundary regularity - free boundary problems.
\end{abstract}

Keywords: boundary Harnack inequality, $p$ harmonic function, Lipschitz domain, Martin boundary, free boundary.

1. Introduction. Denote points in Euclidean $n$ space $\mathbb{R}^{n}$ by $x=\left(x_{1}, \ldots, x_{n}\right)$ or $\left(x^{\prime}, x_{n}\right)$ where $x^{\prime}=\left(x_{1}, \ldots, x_{n-1}\right) \in \mathbb{R}^{n-1}$. Let $\bar{E}, \partial E$, diam $E$, be the closure, boundary, diameter, of the set $E \subset \mathbb{R}^{n}$ and put $d(y, E)$ equal to the distance from $y \in \mathbb{R}^{n}$ to $E$. Let $\langle\cdot, \cdot\rangle$ denote the standard inner product on $\mathbb{R}^{n}$ and let $|x|=\langle x, x\rangle^{1 / 2}$ be the Euclidean norm of $x$. Set $B(x, r)=\left\{y \in \mathbb{R}^{n}:|x-y|<r\right\}$ whenever $x \in \mathbb{R}^{n}, r>0$, and let $d x$ denote Lebesgue $n$ measure on $\mathbb{R}^{n}$. If $O \subset \mathbb{R}^{n}$ is open and $1 \leq q \leq \infty$, we denote by $W^{1, q}(O)$, the space of equivalence classes of functions $f$ with distributional gradient $\nabla f=\left(f_{x_{1}}, \ldots, f_{x_{n}}\right)$, both of which are $q$ th power integrable on $O$. Let $\|f\|_{1, q}=\|f\|_{q}+\||\nabla f|\|_{q}$ be the norm in $W^{1, q}(O)$ where $\|\cdot\|_{q}$ denotes the usual Lebesgue $q$ norm in $O$. Next let $C_{0}^{\infty}(O)$ be infinitely differentiable functions with compact support in $O$ and let $W_{0}^{1, q}(O)$

Received Dec 18, 2006.

2000 Mathematics Subject Classification. Primary 35J25, 35J70

${ }^{*}$ Lewis was partially supported by NSF grant DMS -055228 
be the closure of $C_{0}^{\infty}(O)$ in the norm of $W^{1, q}(O)$. Given $\hat{O}$ a bounded domain (i.e, a connected open set) and $1<p<\infty$, we say that $\hat{u}$ is $p$ harmonic in $\hat{O}$ provided $\hat{u} \in W^{1, p}(\hat{O})$ and

$$
\int|\nabla \hat{u}|^{p-2}\langle\nabla \hat{u}, \nabla \theta\rangle d x=0
$$

whenever $\theta \in W_{0}^{1, p}(\hat{O})$. Observe that if $\hat{u}$ is smooth and $\nabla \hat{u} \neq 0$ in $\hat{O}$, then

$$
\nabla \cdot\left(|\nabla \hat{u}|^{p-2} \nabla \hat{u}\right) \equiv 0 \text { in } \hat{O}
$$

so $\hat{u}$ is a classical solution in $\hat{O}$ to the $p$ Laplace partial differential equation. Here, as in the sequel, $\nabla$. is the divergence operator. We note that $\phi: E \rightarrow \mathbb{R}$ is said to be Lipschitz on $E$ provided there exists $b, 0<b<\infty$, such that

$$
|\phi(z)-\phi(w)| \leq b|z-w| \text {, whenever } z, w \in E .
$$

The infimum of all $b$ such that (1.3) holds is called the Lipschitz norm of $\phi$ on $E$, denoted $\| \phi \hat{\|}_{E}$. It is well known that if $E=\mathbb{R}^{n-1}$, then $\phi$ is differentiable on $\mathbb{R}^{n-1}$ and $\left\|\phi \hat{\|}_{\mathbb{R}^{n-1}}=\right\||\nabla \phi| \|_{\infty}$. Finally let $e_{i}, 1 \leq i \leq n$, denote the point in $\mathbb{R}^{n}$ with one in the $i$ th coordinate position and zeroes elsewhere.

In $[\mathrm{LN}]$ we proved,

Theorem A. Let $G=\left\{y=\left(y^{\prime}, y_{n}\right) \in \mathbb{R}^{n}: y_{n}>\phi\left(y^{\prime}\right)\right\}$ where $\phi$ is Lipschitz on $\mathbb{R}^{n-1}$. Given $p, 1<p<\infty, w=\left(w^{\prime}, \phi\left(w^{\prime}\right)\right) \in \partial G$, and $r>0$, suppose that $u, v$ are positive $p$ harmonic functions in $B(w, r) \cap G$, that $u, v$ are continuous in $\bar{B}(w, r) \cap \bar{G}, u\left(w+\frac{r}{4} e_{n}\right)=v\left(w+\frac{r}{4} e_{n}\right)=1$ and that $u, v=0$ on $B(w, r) \cap \partial G$. Then there exist $c_{1}, 1 \leq c_{1}<\infty$, depending only on $p, n$, and $\||\nabla \phi|\|_{\infty}$ such that

$$
\frac{u(y)}{v(y)} \leq c_{1} \text { whenever } y \in B\left(w, r / c_{1}\right) \cap G \text {. }
$$

In this note we first outline a proof of the following theorem.

Theorem 1. Let $p, \phi, G, u, v, w, r$ be as in Theorem A. Under these assumptions there exists $c_{2}, \quad 1 \leq c_{2}<\infty$, and $\alpha, 0<\alpha<1$, depending only on $p, n$, and $\||\nabla \phi|\|_{\infty}$ such that

$$
\left|\log \frac{u\left(y_{1}\right)}{v\left(y_{1}\right)}-\log \frac{u\left(y_{2}\right)}{v\left(y_{2}\right)}\right| \leq c_{2}\left(\frac{\left|y_{1}-y_{2}\right|}{r}\right)^{\alpha}
$$

whenever $y_{1}, y_{2} \in B\left(w, r / c_{2}\right) \cap G$. 
We note that Theorem 1 implies Theorem A and Hölder continuity of $u / v$ :

$$
\left|\frac{u\left(y_{1}\right)}{v\left(y_{1}\right)}-\frac{u\left(y_{2}\right)}{v\left(y_{2}\right)}\right| \leq c\left(\frac{\left|y_{1}-y_{2}\right|}{r}\right)^{\alpha} \text { for } y_{1}, y_{2} \in B\left(w, r / c_{2}\right) \cap G,
$$

where $c$ has the same dependence as $c_{2}$. Theorem 1 was proved in [JK] for harmonic functions in a nontangentially accessible (NTA) domain. However their proof depends heavily on the fact that the Laplacian is a linear operator, which is not true for the $p$ Laplace operator when $p \neq 2$. The proofs of Theorem A and Theorem 1 overcome this difficulty by considering a certain linear operator for which linear estimates can be used. More specifically if $\hat{u}(\cdot, \tau), \tau \in[0,1]$, is $p$ harmonic in a domain $\hat{O}, \nabla \hat{u}(x, \tau)$ is nonzero for $x \in \hat{O}$, and $\hat{u}$ is sufficiently smooth in $x, \tau$, then $\zeta=\frac{\partial \hat{u}}{\partial \tau}(\cdot, \tau)$ satisfies at $x$ the partial differential equation

$$
L \zeta=\nabla \cdot\left[(p-2)|\nabla \hat{u}|^{p-4}\langle\nabla \hat{u}, \nabla \zeta\rangle \nabla \hat{u}+|\nabla \hat{u}|^{p-2} \nabla \zeta\right]=0,
$$

as follows from differentiating the $p$ Laplace equation in (1.2) with respect to $\tau$. Here we have written $\nabla \hat{u}$ for $\nabla \hat{u}(\cdot, \tau)$. Clearly,

$$
L \hat{u}(x, \cdot)=(p-1) \nabla \cdot\left[|\nabla \hat{u}|^{p-2} \nabla \hat{u}(x, \cdot)\right]=0 .
$$

(1.5) can be written in the form

$$
L \zeta=\sum_{i, j=1}^{n} \frac{\partial}{\partial x_{i}}\left[b_{i j}(x) \zeta_{x_{j}}(x)\right]=0,
$$

where at $x \in \hat{O}$,

$$
b_{i j}(x)=|\nabla \hat{u}|^{p-4}\left[(p-2) \hat{u}_{x_{i}} \hat{u}_{x_{j}}+\delta_{i j}|\nabla \hat{u}|^{2}\right](x), 1 \leq i, j \leq n,
$$

and $\delta_{i j}$ is the Kronecker $\delta$. Thus the first key observation in the proofs of Theorem 1 and Theorem $\mathrm{A}$ is that $\hat{u}(\cdot, \tau)$ and $\frac{\partial \hat{u}}{\partial \tau}(\cdot, \tau)$ both satisfy the divergence form partial differential equation (1.7).

In $[\mathrm{LN}]$ we used this observation, to study deformations of $p$ capacitary functions from one starlike Lipschitz ring to another. More specifically, a bounded domain $\Omega \subset \mathbb{R}^{n}$ is said to be starlike Lipschitz with respect to $\hat{x} \in \Omega$ provided

$$
\partial \Omega=\{\hat{x}+R(\omega) \omega: \omega \in \partial B(0,1)\}
$$

where $\log R: \partial B(0,1) \rightarrow \mathbb{R}$ is Lipschitz on $\partial B(0,1)$. We say that $D$ is a starlike Lipschitz ring domain with center $\hat{x}$ provided $D=\Omega \backslash \bar{\Omega}^{\prime}$ where $\Omega, \Omega^{\prime}$ are starlike Lipschitz domains with center $\hat{x}$ and $\bar{\Omega}^{\prime} \subset \Omega$. Let $R, R^{\prime}$ be the graph functions for $\partial \Omega, \partial \Omega^{\prime}$. As in $[\mathrm{LN}]$ we shall refer to $\left\|\log R \hat{\|}_{\partial B(0,1)}+\right\| \log R^{\prime} \hat{\|}_{\partial B(0,1)}$ as the 
Lipschitz constant for $D$. If $p$ is fixed, $1<p<\infty$, let $\hat{u}=\hat{u}(\cdot, p)$ be the $p$ capacitary function for $D$. That is $\hat{u} \equiv 1$ on $\partial \Omega^{\prime}, \hat{u} \equiv 0$ on $\partial \Omega$ in the sense of $W_{0}^{1, p}(\Omega)$ and $\hat{u}$ is $p$ harmonic in $D$. To simplify the description of our deformations, suppose $\hat{u}_{i}, i=1,2$, are the $p$ capacitary functions for starlike Lipschitz ring domains, $\hat{D}_{i}$ with $\hat{D}_{i}=\hat{\Omega}_{i} \backslash \bar{B}(\hat{x}, \rho)$, where

$$
d\left(\hat{x}, \partial \hat{\Omega}_{1}\right) / 4 \leq \rho \leq d\left(\hat{x}, \partial \hat{\Omega}_{1}\right) / 2 .
$$

Also suppose $\hat{\Omega}_{1} \subset \hat{\Omega}_{2}, w \in \partial \hat{\Omega}_{1} \cap \partial \hat{\Omega}_{2}$, and

$$
B(w, 2 r) \cap \hat{D}_{1}=B(w, 2 r) \cap \hat{D}_{2}
$$

for some fixed $r, 0<r<\rho / 8$. Let $\hat{R}_{i}, i=1,2$, be the corresponding graph functions for $\partial \hat{\Omega}_{i}$ and assume that $\hat{R}_{i}, i=1,2$, are infinitely differentiable on the manifold $\partial B(0,1)$. Put $\hat{R}(\tau)=\hat{R}_{2}^{\tau} \hat{R}_{1}^{1-\tau}, 0 \leq \tau \leq 1$, and let $\hat{\Omega}(\tau)$ be the starlike Lipschitz domain with center $\hat{x}$, graph function $\hat{R}(\tau)$, while $\hat{D}(\tau)=\hat{\Omega}(\tau) \backslash \bar{B}(\hat{x}, \rho)$ is the corresponding ring domain. Let $\hat{u}(\cdot, \tau), \tau \in[0,1]$, be the $p$ capacitary function for $\hat{D}(\tau)$ so that $\hat{u}(\cdot, 0)=\hat{u}_{1}, \hat{u}(\cdot, 1)=\hat{u}_{2}$. In [LN] we showed that $\hat{u}(x, \tau)$ is smooth in $x, \tau$ whenever $x \in \hat{D}_{1}$ and that

$$
|\nabla \hat{u}(x, \tau)| \approx \frac{\hat{u}(x, \tau)}{d(x, \partial \hat{\Omega}(\tau))} \approx\left\langle\frac{\hat{x}-x}{\hat{x}-x \mid}, \nabla \hat{u}(x, \tau)\right\rangle \text { whenever } x \in \hat{D}(\tau) .
$$

Moreover, $\hat{u}(x, \tau), \tau \in[0,1]$, provides a smooth deformation of $\hat{u}_{1}(x)$ to $\hat{u}_{2}(x)$ and so (1.5)-(1.8) are true. It follows from this deduction that,

$$
\log \left(\frac{\hat{u}_{2}(x)}{\hat{u}_{1}(x)}\right)=\int_{0}^{1} \frac{\hat{u}_{\tau}(x, \tau)}{\hat{u}(x, \tau)} d \tau
$$

From (1.10), (1.5)-(1.8), one also sees that $u_{\tau} \geq 0$ and $u_{\tau} \equiv 0$ on $B(w, 2 r) \cap \partial \hat{D}(\tau)$. In [LN, Theorem 2] we used these observations, (1.5) - (1.11), and some deep results on elliptic measure to conclude that $\hat{u}_{\tau}(\cdot, \tau) / \hat{u}(\cdot, \tau)$ is Hölder continuous in $B(w, r) \cap \hat{D}_{1}$ with constants independent of $\tau \in[0,1]$. From this conclusion and (1.12) it easily follows that Theorem 1 holds with $u, v$ replaced by $\hat{u}_{1}, \hat{u}_{2}$. In fact in [LN, Theorem 2] we prove (1.4) for $\hat{u}_{1}, \hat{u}_{2}$, but we were not able to prove (1.4) in general, primarily because we did not see how to define a deformation $\{u(\cdot, \tau)\}$ of $u$ into $v$ for which $(1.11$ ) held (with $\hat{u}(\cdot, \tau)$ replaced by $u(\cdot, \tau)$ ). Furthermore without (1.11) we could not prove that $u_{\tau} / u$ is Hölder continuous.

In this paper, after some preliminary reductions, we let $u(\cdot, \tau), \tau \in[0,1]$, be the $p$ harmonic function in $B\left(w, 2 r^{\prime}\right) \cap G$ with boundary values $u(\cdot, \tau)=(1-\tau) u+\tau v$ on $\partial\left[B\left(w, 2 r^{\prime}\right) \cap G\right]$. Here $r^{\prime}=\frac{r}{4 c_{1}^{2}}$ where $c_{1}$ is as in Theorem A. We then show 
that (1.11) and Theorem 1 hold for this deformation in $B(w, r / \hat{c}) \cap G$, if $\hat{c}$ is large enough. The proof uses a method of continuity - iteration type argument. That is, we first show (1.11) holds for $\tau \in\left[0, \epsilon_{1}\right]$ whenever $x \in B\left(w, r^{\prime} / c\right) \cap G$ for some small $\epsilon_{1}>0$. We then use the techniques from [LN] to get Theorem 1 with $v$ replaced by $u\left(\cdot, \epsilon_{1}\right)$. Second we use Theorem 1 for $u\left(\cdot, \epsilon_{1}\right)$ to get $(1.11)$ for $u(\cdot, \tau)$ on $B\left(w, r^{\prime} / c^{\prime}\right) \cap G, c^{\prime}>c$, when $\tau \in\left[\epsilon_{1}, 2 \epsilon_{1}\right]$. Next we prove Hölder continuity of $u\left(\cdot, 2 \epsilon_{1}\right)$ in $B\left(w, r^{\prime} / c^{*}\right) \cap G, c^{*}>c^{\prime}$, and then use this result to show that (1.11) holds for $\tau \in\left[2 \epsilon_{1}, 3 \epsilon_{1}\right]$ on $B\left(w, r^{\prime} / c^{* *}\right) \cap G, c^{* *}>c^{*}$. Continuing by induction we eventually get Theorem 1. An important part of the proof is to show that $\epsilon_{1}$ can be chosen to depend only on $p, n$, and $\||\nabla \phi|\|_{\infty}$.

As for the plan of this paper, in section 2 we give some basic estimates for $p$ harmonic functions and state some results from [LN] which will be used in the proof of Theorem 1. In section 3 we prove Theorem 1. In section 4 we discuss some applications of Theorem 1 to the Martin boundary problem given in [LN1]. In section 5 we outline some applications of Theorem 1 to boundary value problems given in [LN2]. Finally we remark that for the reader well versed in [LN] our proof of Theorem 1 is essentially self contained.

2. Basic Estimates and Results from [LN]. Let $G, w, r$ be as in Theorem $\mathrm{A}$ and suppose that for some fixed $p, 1<p<\infty, \hat{u}>0$ is $p$ harmonic in $B(w, \tilde{r}) \cap G, 0<\tilde{r} \leq r$, continuous on $\bar{B}(w, \tilde{r}) \cap G$ and $\hat{u} \equiv 0$ on $B(w, \tilde{r}) \cap G$. Put $\hat{u}=0$ on $B(w, \tilde{r}) \backslash \bar{G}$. With $\hat{u}$ now defined on $B(w, \tilde{r})$ let $\max _{B(z, s)} \hat{u}, \min _{B(z, s)} \hat{u}$ be the maximum and minimum of $\hat{u}$ on $B(z, s)$ whenever $\bar{B}(z, s) \subset B(w, \tilde{r})$. We begin this section by stating some interior and boundary estimates for $\hat{u}$.

Lemma 2.1. Let $\hat{u}$ be as above. If $\bar{B}(y, 2 s) \subset B(w, \tilde{r})$, then

$$
s^{p-n} \int_{B(y, s / 2)}|\nabla \hat{u}|^{p} d x \leq c \max _{B(y, s)} \hat{u}^{p}
$$

while if $B(y, 2 s) \subset B(w, \tilde{r}) \cap G$, then

$$
\max _{B(y, s)} \hat{u} \leq c \min _{B(y, s)} \hat{u} .
$$

If $y \in B(w, \tilde{r} / 2) \cap \partial G, B(y, 2 s) \subset B(w, \tilde{r})$, and $s \leq \tilde{r} / c$, then

$$
\max _{B(y, s)} \hat{u} \leq c \hat{u}\left(a_{s}(y)\right)
$$


where $a_{s}(y)$ is a point in $\bar{B}(y, s) \cap G$ with $d\left(a_{s}(y), \partial G\right)=\sup \{d(z, \partial G): z \in$ $\bar{B}(y, s) \cap G\}$. Moreover, there exists $\alpha, 0<\alpha<1$, and $c$ such that if $B(y, 2 s) \subset$ $B(w, \tilde{r})$, then

$$
|\hat{u}(x)-\hat{u}(z)| \leq c\left(\frac{|x-z|}{s}\right)^{\alpha} \max _{B(y, 2 s)} \hat{u} \text { whenever } x, z \in B(y, s) .
$$

Proof: (2.2) is a standard subsolution estimate. (2.3) is a well known Harnack inequality for positive solutions of $p$ Laplacian type. (2.4) is due to Carleson Domar. If $B(y, 2 s) \subset B(w, \tilde{r}) \cap G$, then (2.5) is a well known interior Hölder continuity estimate for solutions of $p$ Laplacian type. Otherwise, (2.5) follows from simple barrier type estimates and interior Hölder continuity (references for the proofs of (2.2)-(2.5) are given in [LN]).

In Lemma 2.1, as well as in the sequel, $c$ denotes a positive constant, not necessarily the same at each occurrence, which may depend only on $p, n$, and $\||\nabla \phi|\|_{\infty}$. In general $c\left(a_{1}, a_{2}, \ldots, a_{m}\right)$ denotes a positive constant which may only depend on $p, n,\||\nabla \phi|\|_{\infty}$, and $a_{1}, \ldots, a_{m}$. We note that $\alpha$ in Lemma 2.1 depends only on $p, n$, and $\||\nabla \phi|\|_{\infty}$.

Lemma 2.6. $\hat{u}$ has a representative in $W^{1, p}(B(w, \tilde{r}))$ that has Hölder continuous partial derivatives in $B(w, \tilde{r}) \cap G$. That is, for some $\sigma \in(0,1]$ (depending only on $p, n)$ we have

(2.7) $c^{-1}|\nabla \hat{u}(x)-\nabla \hat{u}(z)| \leq(|x-z| / s)^{\sigma} \max _{B(y, s / 2)}|\nabla \hat{u}| \leq c s^{-1}(|x-z| / s)^{\sigma} \hat{u}(y)$ whenever $x, z \in B(y, s / 4)$ and $B(y, 2 s) \subset G$. Also, if $c|\nabla \hat{u}(x)| \geq \hat{u}(x) / d(x, \partial G)$ for all $x \in B(y, 2 s)$, then

$$
\begin{aligned}
\max _{B\left(y, \frac{s}{4}\right)} \sum_{i, j=1}^{n}\left|\hat{u}_{x_{i} x_{j}}\right| & \leq c\left(s^{-n} \int_{B(y, s / 2)} \sum_{i, j=1}^{n}\left|\hat{u}_{x_{i} x_{j}}\right|^{2} d y\right)^{1 / 2} \\
& \leq c^{2} \hat{u}(y) / d(y, \partial G)^{2} .
\end{aligned}
$$

Moreover if $\nabla \hat{u}(x) \neq 0$, then $\hat{u}$ is $C^{\infty}$ in a neighborhood of $x$.

Proof: See [LN, Lemmas 2.3, 2.4, 2.5 ]. 
Lemma 2.9. If $\phi \in C_{0}^{\infty}\left(\mathbb{R}^{n-1}\right)$ (i.e $\partial G$ is $\left.C^{\infty}\right)$, then there exists an open neighborhood $N \supset B(w, \tilde{r} / 2) \cap \partial G$ such that $\hat{u}$ has a $C^{\infty}$ extension to the closure of $N \cap G \cap B(w, \tilde{r} / 2)$. Furthermore, there exists $b \geq 1, \beta, 0<\beta<1$, depending only on $p, n$, and the $C^{3}$ norm of $\phi$ such that

(a) $\quad b^{-1} \hat{u}(y) / d(y, \partial G) \leq|\nabla \hat{u}(y)| \leq b \hat{u}(y) / d(y, \partial G)$ whenever $y \in N \cap G \cap B(w, \tilde{r} / 2)$,

(b) $|\nabla \hat{u}(x)-\nabla \hat{u}(z)| \leq b(|x-z| / s)^{\beta} \hat{u}(y) / d(y, \partial G)$ if $y \in B(w, \tilde{r} / 2) \cap G, B(y, 2 d(y, \partial G)) \subset N$, and $x, z \in B(y, 2 d(y, \partial G))$.

Proof: See Lemma 2.4 in [LN].

Next suppose for fixed $p, 1<p<\infty$, that $\tilde{u}$ is the $p$ capacitary for the starlike Lipschitz ring domain $\tilde{D}=\tilde{\Omega} \backslash B(\hat{x}, \rho)$ where $c^{-1} d(\hat{x}, \partial \tilde{D}) \leq \rho \leq \frac{1}{4} d(\hat{x}, \partial \tilde{D})$.

Lemma 2.11. Let $\tilde{u}, \tilde{D}, p$, be as above. There exists $c$ depending only on $p, n$, and the Lipschitz constant such that

(i) $\quad 0<|\nabla \tilde{u}(x)| \leq c\left\langle\frac{\hat{x}-x}{|\hat{x}-x|}, \nabla \tilde{u}(x)\right\rangle$ whenever $x \in \tilde{D}$.

(ii) $\quad c^{-1} \tilde{u}(x) / d(x, \partial \tilde{D}) \leq|\nabla \tilde{u}(x)| \leq c \tilde{u}(x) / d(x, \partial \tilde{D})$ whenever $x \in \tilde{D}$.

Proof: See Lemma 2.5 in [LN].

Let $\tilde{u}$ be as in Lemma 2.11 and define $L, b_{i j}$ as in (1.7), (1.8) with $\hat{u}$ replaced by $\tilde{u}$. In [LN] we use Lemmas 2.1, 2.6, 2.9, 2.11, to prove Lemmas 3.13 and 3.27. Together they are equivalent to the following lemma.

Lemma 2.13. Let $y \in \partial \tilde{\Omega}, 0<s<\rho / 8$, and suppose that $\partial \tilde{D}$ is $C^{\infty}$. Let $h_{1}, h_{2}$ be positive solutions to $L$ in $B(y, s) \cap \tilde{D}$ with $h_{i}=0, i=1,2$, continuously on $B(y, s) \cap \partial \tilde{D}$. There exists $\hat{c} \geq 1, \lambda, 0<\lambda<1$ (depending only on $p, n$ and the 
Lipschitz constant for $\tilde{D}$ ) such that if $\hat{s}=s / \hat{c}$, then

$$
\left|\log \frac{h_{1}\left(z_{1}\right)}{h_{2}\left(z_{1}\right)}-\log \frac{h_{1}\left(z_{2}\right)}{h_{2}\left(z_{2}\right)}\right| \leq \hat{c}\left(\frac{\left|z_{1}-z_{2}\right|}{s}\right)^{\lambda}
$$

whenever $z_{1}, z_{2} \in B(y, \hat{s}) \cap \tilde{D}$.

Proof: We here briefly outline the proof in [LN, Lemma 2.39] of Lemma 2.13. We first use the above lemmas and some Rellich type inequalities to show, as in [G], the existence of $q>p$, depending only on $p, n$, and the Lipschitz constant for $\tilde{D}$, such that the following reverse Hölder inequality holds,

$$
\begin{aligned}
& \int_{B(y, s) \cap \partial \tilde{\Omega}}|\nabla \tilde{u}|^{q} d H^{n-1} \\
& \leq c s^{(n-1)\left(\frac{p-1-q}{p-1}\right)}\left(\int_{B(y, s) \cap \partial \tilde{\Omega}}|\nabla \tilde{u}|^{p-1} d H^{n-1}\right)^{q /(p-1)} .
\end{aligned}
$$

Here $y, s$ are as in Lemma 2.13 and $H^{n-1}$ denotes Hausdorff $n-1$ measure on $\partial \tilde{\Omega}$. Let $\tilde{y} \in \tilde{D}$ be the point on the ray from $\hat{x}$ to $y$ with $|\tilde{y}-y|=s / 4$. Using (2.15), we show in [LN, Lemma 2.45] that there exists a starlike Lipschitz domain $\Omega^{*} \subset B(y, s) \cap \tilde{\Omega}$ with center $\tilde{y}$ satisfying

$$
\begin{aligned}
& \text { (a) } \quad c H^{n-1}\left[\partial \tilde{\Omega} \cap B(y, s) \cap \partial \Omega^{*}\right] \geq s^{n-1} \\
& \text { (b) } \quad c^{-1} s^{-1} \tilde{u}(\tilde{y}) \leq|\nabla \tilde{u}(x)| \leq c s^{-1} \tilde{u}(\tilde{y}) \text { whenever } x \in \Omega^{*} .
\end{aligned}
$$

Next in [LN, Lemma 2.54] we define

$$
d \tilde{\sigma}(x)=d\left(x, \partial \Omega^{*}\right) \max _{B\left(x, \frac{1}{2} d\left(x, \partial \Omega^{*}\right)\right)}\left\{|\nabla \tilde{u}|^{2 p-6} \sum_{i, j=1}^{n} \tilde{u}_{x_{i} x_{j}}^{2}\right\} d y
$$

when $x \in \Omega^{*}$, and use (2.16), (2.8) to show that $\tilde{\sigma}$ is a Carleson measure on $\Omega^{*}$ in the sense that if $z \in \partial \Omega^{*}$ and $0<t<s / 4$, then

$$
\tilde{\sigma}\left(B(z, t) \cap \Omega^{*}\right) \leq c t^{n-1}(\tilde{u}(\tilde{y}) / s)^{2 p-4} .
$$

Armed with (2.17) we can apply a theorem in $[\mathrm{KP}]$ to deduce that if $\omega^{*}$ is elliptic measure defined with respect to $L,\left(b_{i j}\right)$ in $\Omega^{*}$, then $\omega^{*}$ is an $A^{\infty}$ weight with respect to $H^{n-1}$ measure on $\partial \Omega^{*}$ (see [LN, Theorem 3.11]). Finally in [LN], we use this result for $\omega^{*}$ as well as some comparison arguments for elliptic measure to get Lemma 2.13 . 
Remark: It is important to note that Lemma 2.13 is valid whenever $\tilde{u}$ satisfies the hypotheses of Lemmas 2.1, 2.6, 2.9, 2.11 and does not depend on the fact that $\tilde{u}$ is a $p$ capacitary function. Moreover, Lemmas 2.1, 2.6, 2.9 are standard estimates for a positive $p$ harmonic function vanishing on a portion of the boundary of a Lipschitz domain. Thus Lemma 2.11 is the only lemma which requires proof (for the positive $p$ harmonic functions we consider) in order to use Lemma 2.13 .

3. Proof of Theorem 1. To begin the proof of Theorem 1, put $u=v=0$ in $B(w, r) \backslash G$. Then $u, v$ are continuous in $B(w, r)$. We assume, as we may, that

$$
\phi \in C_{0}^{\infty}\left(\mathbb{R}^{n-1}\right)
$$

Indeed otherwise let $G_{\epsilon}$ be the domain defined as in Theorem A with $\phi$ replaced by $\phi_{\epsilon}$ where $\phi-\epsilon<\phi_{\epsilon}<\phi$ in $\left\{x^{\prime} \in \mathbb{R}^{n-1}:\left|x^{\prime}-w^{\prime}\right|<2 r\right\}, \phi_{\epsilon} \in C^{\infty}\left(\mathbb{R}^{n}\right)$, and $\left\|\left|\nabla \phi_{\epsilon}\right|\right\|_{\infty} \leq\||\nabla \phi|\|_{\infty}$. Existence of $\phi_{\epsilon}$ follows from convoluting $\phi-\epsilon / 2$ with a suitable approximate identity. Let $u_{\epsilon}, v_{\epsilon}$, be the unique positive $p$ harmonic functions in $B(w, r / 2) \cap G_{\epsilon}$ with $u_{\epsilon}=u, v_{\epsilon}=v$ on $\partial\left[B(w, r / 2) \cap G_{\epsilon}\right]$ in the $W^{1, p}$ Sobolev sense. Existence and uniqueness of $u_{\epsilon}, v_{\epsilon}$ follow from well known arguments in the calculus of variations. From our choice of $\phi_{\epsilon}$ we have $u_{\epsilon}=v_{\epsilon}=0$ on $B(w, r / 2) \cap \partial G_{\epsilon}$ in the $W_{0}^{1, p}$ Sobolev sense. Now from Lemma 2.1 it is easily seen that $u_{\epsilon}, v_{\epsilon}$ converge uniformly to $u, v$ on compact subsets of $B(w, r / 2) \cap G$ as $\epsilon \rightarrow 0$. Since $c_{2}$ in Theorem 1 depends only on $p, n$ and the Lipschitz norm of $\nabla \phi_{\epsilon}$, it follows that we can prove Theorem 1 for $u_{\epsilon}, v_{\epsilon}$, and then take limits to get Theorem 1 for $u, v$. Thus we assume (3.1).

Next let $\hat{x}=w+\frac{1}{4} r e_{n}$ and observe from basic geometry that if $c^{\prime}$ is large enough (depending on the Lipschitz norm of $\phi$ ), then the domain $\tilde{\Omega} \subset G$ obtained from drawing all open line segments from points in $B\left(w, r / c^{\prime}\right) \cap \partial G$ to points in $B\left(\hat{x}, r / c^{\prime}\right)$ is starlike Lipschitz with center $\hat{x}$ and Lipschitz constant $\leq c\left(\||\nabla \phi|\|_{\infty}+1\right)$, where $c$ depends only on $n$. Let $\rho=\frac{r}{16 c^{\prime}}$ and let $\tilde{u}$ be the $p$ capacitary function for $\tilde{D}=\tilde{\Omega} \backslash \bar{B}(\hat{x}, \rho)$. Then $\tilde{D}$ is a starlike ring domain and from Lemma 2.11 we see that (2.12) is valid for $\tilde{u}$. To continue note that if $c_{1}$ in Theorem A is large, then we can also assume

$$
B\left(w, r / c_{1}\right) \cap \partial G=B\left(w, r / c_{1}\right) \cap \partial \tilde{D} .
$$


From (3.2), arbitrariness of $v$ and the triangle inequality, we find that it suffices to prove Theorem 1 with $u$ replaced by $\tilde{u}$, and $r$ by $r / c_{1}$ provided $c_{1}$ is large enough. Thus we write $u$ for $\tilde{u}$ and assume $u$ satisfies (2.12). We also assume for for technical reasons that

$$
u \leq v / 2 \leq c_{3} u \text { in } \bar{B}\left(w, r / c_{1}^{2}\right) \cap G
$$

where $c_{3}$ has the same dependence as $c_{1}$. Otherwise, we can multiply $v$ by a positive constant to get this inequality, thanks to Theorem A with $r$ replaced by $r / c_{1}$. Let $r^{\prime}=\frac{r}{4 c_{1}^{2}}$ and let $u(\cdot, \tau), 0 \leq \tau \leq 1$, be the $p$ harmonic function in $B\left(w, 2 r^{\prime}\right) \cap G$ with

$$
u(\cdot, \tau)=\tau v+(1-\tau) u
$$

on $\partial\left[B\left(w, 2 r^{\prime}\right) \cap G\right]$ for $0 \leq \tau \leq 1$. Next we prove a key lemma.

Lemma 3.5. There exists, $\epsilon_{0} \in(0,1 / 4)$, depending only on $p, n$, and $\||\nabla \phi|\|_{\infty}$ such that if for some $\hat{\tau} \in[0,1], \hat{L}, 0<\hat{L}<\infty$, and $s, 0<s \leq r^{\prime}$ we have

$$
\left(1-\epsilon_{0}\right) \hat{L} \leq \frac{u(\cdot, \hat{\tau})}{u} \leq\left(1+\epsilon_{0}\right) \hat{L} \text { in } B(w, s),
$$

then for some $c_{4} \geq 1$, having the same dependence as $c_{1}$,

$$
c_{4}\left\langle\frac{\hat{x}-x}{|\hat{x}-x|}, \nabla u(x, \hat{\tau})\right\rangle \geq u(x, \hat{\tau}) / d(x, \partial G) \text { whenever } x \in B(w, s / 4) \cap G .
$$

Proof: Let $x \in B(w, s / 4) \cap G$. From (2.7) with $\hat{u}$ replaced by $u$ we see that

$$
\begin{aligned}
\left|\nabla u\left(z_{1}, \hat{\tau}\right)-\nabla u\left(z_{2}, \hat{\tau}\right)\right| & \leq c t^{\sigma} \max _{B(x, 2 t d(x, \partial G))}|\nabla u(\cdot, \hat{\tau})| \\
& \leq c^{2} t^{\sigma} u(x, \hat{\tau}) / d(x, \partial G)
\end{aligned}
$$

whenever $z_{1}, z_{2} \in \bar{B}(x, t d(x, \partial G))$ and $0<t \leq 1 / 4$. Here $c$ depends only on $p, n$. Now suppose that

(3.9) $\langle\nabla u(x, \hat{\tau}), \omega\rangle \leq \eta u(x, \hat{\tau}) / d(x, \partial G)$ for some $\eta>0$ and $x \in B(w, s / 4) \cap G$ where $\omega=\frac{\hat{x}-x}{|\hat{x}-x|}$. From (3.8) with $z=z_{1}, x=z_{2}$ and (3.9) we deduce

$$
\langle\nabla u(z, \hat{\tau}), \omega\rangle \leq\left[\eta+c^{2} t^{\sigma}\right] u(x, \hat{\tau}) / d(x, \partial G)
$$

for $z \in B(x, t d(x, \partial G))$. Integrating, it follows that if $y=x+t d(x, \partial G) \omega$, and $t=\eta^{1 / \sigma}$, then

$$
u(y, \hat{\tau})-u(x, \hat{\tau}) \leq c^{\prime} \eta^{1+1 / \sigma} u(x, \hat{\tau})
$$


In (3.11) $c^{\prime}$ depends only on $p, n$. Now from (2.12) and the mean value theorem, we also deduce for $z$ as above and for some $c^{*}$ (depending only on $p, n,\||\nabla \phi|\|_{\infty}$ ), that

$$
c^{*}(u(y)-u(x)) \geq \eta^{1 / \sigma} u(x)
$$

Note that if $\epsilon_{0}, \hat{L}$ are as in (3.6), then from (3.11), (3.12) we find that

$$
\begin{gathered}
\left(1-\epsilon_{0}\right) \hat{L} \leq \frac{u(y, \hat{\tau})}{u(y)} \leq\left(\frac{1+c^{\prime} \eta^{1+1 / \sigma}}{1+\eta^{1 / \sigma} / c^{*}}\right) \frac{u(x, \hat{\tau})}{u(x)} \\
\quad \leq\left(1+\epsilon_{0}\right)\left(\frac{1+c^{\prime} \eta^{1+1 / \sigma}}{1+\eta^{1 / \sigma} / c^{*}}\right) \hat{L}<\left(1-\epsilon_{0}\right) \hat{L}
\end{gathered}
$$

provided $1 / \tilde{c} \geq \eta^{1 / \sigma} \geq \tilde{c} \epsilon_{0}$ for some large $\tilde{c}$ with the same dependence as $c_{1}$. With $\tilde{c}$ now fixed we put $\epsilon_{0}=1 / \tilde{c}^{2}$ and assume that the hypotheses of Lemma 3.5 hold for this $\epsilon_{0}$. Then in order to avoid the contradiction in (3.13), it must be true that

$$
\langle\nabla u(x, \hat{\tau}), \omega\rangle \geq \frac{u(x, \hat{\tau})}{\tilde{c}^{\sigma} d(x, \partial G)} \text { for } x \in B(w, s / 4) \cap G .
$$

Thus (3.7) holds and Lemma 3.5 is true.

To continue the proof of Theorem 1 , observe from (3.3) that if $\tau_{1}, \tau_{2} \in[0,1]$ then

$$
c^{-1} u\left(\cdot, \tau_{1}\right) \leq \frac{u\left(\cdot, \tau_{2}\right)-u\left(\cdot, \tau_{1}\right)}{\tau_{2}-\tau_{1}}=v-u \leq c u\left(\cdot, \tau_{1}\right)
$$

on $\partial\left(B\left(w, 2 r^{\prime}\right) \cap G\right)$. From the boundary maximum principle for $p$ harmonic functions this inequality also holds in $B\left(w, 2 r^{\prime}\right) \cap G$. Thus for $\epsilon_{0}$ as in Lemma 3.5 there exists $\epsilon_{0}^{\prime}, 0<\epsilon_{0}^{\prime} \leq \epsilon_{0}$, with the same dependence as $\epsilon_{0}$, such that if $\left|\tau_{2}-\tau_{1}\right| \leq \epsilon_{0}^{\prime}$, then

$$
1-\epsilon_{0} / 2 \leq \frac{u\left(\cdot, \tau_{2}\right)}{u\left(\cdot, \tau_{1}\right)} \leq 1+\epsilon_{0} / 2 \text { in } B\left(w, 2 r^{\prime}\right) \cap G .
$$

Divide $[0,1]$ into closed intervals, disjoint except for endpoints, of length $\epsilon_{0}^{\prime} / 2$ except possibly for the interval containing 1 which is of length $\leq \epsilon_{0}^{\prime} / 2$. Let $\xi_{1}=$ $0<\xi_{2}<\ldots<\xi_{m}=1$ be the endpoints of these intervals. Thus $[0,1]$ is divided into $\left\{\left[\xi_{k}, \xi_{k+1}\right]\right\}, 1 \leq k \leq m-1$. Next suppose for some $l, 1 \leq l \leq m-1$, that 
(3.7) is valid with $\hat{\tau}$ replaced by $\tau$ whenever $\tau \in\left[\xi_{l}, \xi_{l+1}\right]$. Under this assumption we claim for some $c_{5}, c_{6}, \alpha$, having the usual dependence, that

$$
\left|\log \frac{u\left(x, \xi_{l+1}\right)}{u\left(x, \xi_{l}\right)}-\log \frac{u\left(y, \xi_{l+1}\right)}{u\left(y, \xi_{l}\right)}\right| \leq c_{5}\left(\frac{|x-y|}{s}\right)^{\alpha}
$$

whenever $x, y \in B\left(w, s / c_{6}\right) \cap G$. Indeed from (3.7) and (2.7) we see that (2.12) holds with $\tilde{u}$ replaced by $u(\cdot, \tau)$ whenever $\tau \in\left[\xi_{l}, \xi_{l+1}\right]$ and for a constant having the same dependence as $c_{1}$ (hence independent of $\tau$ ). To prove claim (3.16) we observe from (3.14), for fixed $x \in B(w, s / 4) \cap G$, that $\tau \rightarrow u(x, \tau), \tau \in[0,1]$, is Lipschitz with norm $\leq c u(x)$. Thus $u_{\tau}(x, \cdot)$ exists almost everywhere in $[0,1]$. Let $\left(x_{\nu}\right)$ be a dense sequence of $B(w, s / 4)$. Let $W$ be the set of all $\tau \in[0,1]$ for which $u_{\tau}\left(x_{m}, \cdot\right)$ exists in the difference quotients sense whenever $x_{m} \in\left(x_{\nu}\right)$. Then $H^{1}([0,1] \backslash W)=0$ where $H^{1}$ is linear Lebesgue or Hausdorff one measure. Let $s, \tau \in\left[\xi_{l}, \xi_{l+1}\right]$. From Lemma 2.9 and $(3.7)$ applied to $u(\cdot, \tau)$ we see as in [LN, (4.6)-(4.8)] that if

$$
a_{i j}(\eta)=|\eta|^{p-4}\left[(p-2) \eta_{i} \eta_{j}+\delta_{i j}|\eta|^{2}\right] \text { for } \eta \in \mathbb{R}^{n} \backslash\{0\}
$$

and

$$
\begin{gathered}
U(x)=U(x, \tau, t)=\frac{u(x, t)-u(x, \tau)}{t-\tau} \\
A_{i j}(x)=A_{i j}(x, \tau, t)=\int_{0}^{1} a_{i j}[\lambda \nabla u(x, t)+(1-\lambda) \nabla u(x, \tau)] d \lambda, 1 \leq i, j \leq n,
\end{gathered}
$$

then, whenever $x \in B(w, s / 4) \cap G$,

$$
\tilde{L} U(x)=\sum_{i, j=1}^{n} \frac{\partial}{\partial x_{i}}\left[A_{i j}(x) U_{x_{j}}\right]=0 \text { on } B(w, s / 4) \cap G .
$$

Moreover, if $x \in B(w, s / 4) \cap G$, then it is easily seen that

$$
\left.(*) \quad c^{-1}|\xi|^{2}|| \nabla u(x, t)|+| \nabla u(x, \tau)\right|^{p-2} \leq \sum_{i, j=1}^{n} A_{i j}(x) \xi_{i} \xi_{j},
$$

$$
(* *) \sum_{i, j=1}^{n}\left|A_{i j}(x)\right| \leq c|| \nabla u(x, t)|+| \nabla u(x, \tau)||^{p-2},
$$

whenever $\xi \in \mathbb{R}^{n} \backslash\{0\}$ and where $c$ depends only on $p, n$. Note that $u(x, t) \rightarrow u(x, \tau)$ uniformly in the closure of $B(x, s / 8) \cap G$ thanks to Lemma 2.1 and (3.15) with $\tau_{1}, \tau_{2}$ replaced by $\tau, t$. From Lemma 2.9, (3.14), (2.12), (3.17) - (3.18), and 
Schauder type arguments we see that $U$ is a bounded (independent of $\tau, t$ ) $C^{\infty}$ solution to a uniformly elliptic PDE in divergence form on $B(w, s / 8) \cap G$ with $U \equiv 0$ on $B(w, s / 8) \cap \partial G$. Put $U \equiv 0$ in $B(w, s / 4) \backslash G$. From Schauder type estimates for this PDE it follows that a subsequence of $\{U(\cdot, \tau, t)\}$, say $\left\{U\left(\cdot, \tau, t_{k}\right)\right\}$ with $t_{k} \rightarrow \tau$ as $k \rightarrow \infty$, converges uniformly to $f=f(\cdot, \tau)$ in $B(w, s / 8)$ with $f \in C^{\infty}[B(w, s / 8) \cap G]$. Moreover,

(a) $f$ is a solution to $(1.7),(1.8)$ in $B(w, s / 8) \cap G$,

(b) $f$ is continuous in $B(w, s / 8)$ with $f \equiv 0$ on $B(w, s / 8) \backslash G$,

(c) $\quad f\left(x_{m}, \tau\right)=u_{\tau}\left(x_{m}, \tau\right)$ when $x_{m} \in B(w, s / 8) \cap G, \tau \in W$,

(d) $\quad c^{-1} \leq f(\cdot, \tau) / u(\cdot, \tau) \leq c$ on $B(w, s / 8) \cap G$.

From (3.19) and (3.7) for $u(\cdot, \tau), \tau \in\left[\xi_{l}, \xi_{l+1}\right]$, we deduce that Lemma 2.13 (see the remark below the proof of Lemma 2.13) can be applied with $h_{1}=u(\cdot, \tau), h_{2}=$ $f(\cdot, \tau)$, whenever $\tau \in\left[\xi_{l}, \xi_{l+1}\right]$. Doing this and using (3.19) (c) we obtain for $x, y \in\left(x_{\nu}\right)$ and $x, y \in B(w, s / c) \cap G$ that

$$
\begin{gathered}
\left|\log \frac{u\left(x, \xi_{l+1}\right)}{u\left(x, \xi_{l}\right)}-\log \frac{u\left(y, \xi_{l+1}\right)}{u\left(y, \xi_{l}\right)}\right|=\left|\int_{\xi_{l}}^{\xi_{l+1}}\left(\frac{f(x, \tau)}{u(x, \tau)}-\frac{f(y, \tau)}{u(y, \tau)}\right) d \tau\right| \\
\leq \int_{\xi_{l}}^{\xi_{l+1}}\left|\frac{f(x, \tau)}{u(x, \tau)}-\frac{f(y, \tau)}{u(y, \tau)}\right| d \tau \leq c\left(\frac{|x-y|}{s}\right)^{\alpha} .
\end{gathered}
$$

From continuity of $u\left(\cdot, \xi_{l+1}\right) / u\left(\cdot, \xi_{l}\right)$ we conclude that (3.20) holds for all $x, y \in$ $B(w, s / c) \cap G$. Thus claim (3.16) is valid.

We now proceed by induction. Observe from our choice of $\epsilon_{0}^{\prime}$ as well as $u\left(\cdot, \xi_{1}\right)=u$ that the hypotheses of Lemma 3.5 are satisfied whenever $\hat{\tau} \in\left[\xi_{1}, \xi_{2}\right]$. Thus (3.16) is true for $l=1$ with $s=r^{\prime}$. Let $r_{2}=r^{\prime} / c_{6}$. Continuing by induction, suppose for some $2 \leq k<m$ that we have shown

$$
\left|\log \frac{u\left(x, \xi_{k}\right)}{u(x)}-\log \frac{u\left(y, \xi_{k}\right)}{u(y)}\right| \leq(k-1) c_{5}\left(\frac{|x-y|}{r_{k}}\right)^{\alpha}
$$

whenever $x, y \in B\left(w, r_{k}\right) \cap G$ where $\alpha, c_{5}$ are the constants in (3.16). Choose $r_{k}^{\prime} \leq r_{k}$ so that

$$
\left|\frac{u\left(x, \xi_{k}\right)}{u(x)}-\frac{u\left(y, \xi_{k}\right)}{u(y)}\right| \leq \delta \frac{u\left(x, \xi_{k}\right)}{u(x)}
$$


whenever $x, y \in B\left(w, r_{k}^{\prime}\right) \cap G$. Fix $x \in B\left(w, r_{k}^{\prime}\right) \cap G$ and choose $\delta>0$ so small that

$$
\left(1-\epsilon_{0}\right) \frac{u\left(x, \xi_{k}\right)}{u(x)} \leq \frac{u(y, \tau)}{u(y)} \leq\left(1+\epsilon_{0}\right) \frac{u\left(x, \xi_{k}\right)}{u(x)}
$$

whenever $y \in B\left(w, r_{k}^{\prime}\right) \cap G$ and $\tau \in\left[\xi_{k}, \xi_{k+1}\right]$. To see the size of $\delta$ observe for $\tau \in\left[\xi_{k}, \xi_{k+1}\right]$ that

$$
\frac{u(y, \tau)}{u(y)}=\frac{u(y, \tau)}{u\left(y, \xi_{k}\right)} \cdot \frac{u\left(y, \xi_{k}\right)}{u(y)} \leq\left(1+\epsilon_{0} / 2\right)(1+\delta) \frac{u\left(x, \xi_{k}\right)}{u(x)} .
$$

Thus if $\delta=\epsilon_{0} / 4$ and if $\epsilon_{0}$ is small enough then the right hand inequality in (3.22) is valid. A similar argument gives the left hand inequality in (3.22) when $\delta=\epsilon_{0} / 4$ and $\epsilon_{0}$ is small enough. Also since $\alpha$ is independent of $k, k \leq 2 / \epsilon_{0}^{\prime}$, and $\epsilon_{0}^{\prime}$ has the usual dependence, we deduce from (3.21) that one can take $r_{k}^{\prime}=r_{k} / c_{7}$ for $c_{7}$ large enough, depending only on $p, n,\||\nabla \phi|\|_{\infty}$. From (3.22) we find that (3.6) holds with $\hat{L}=\frac{u\left(x, \xi_{k}\right)}{u(x)}, s=r_{k}^{\prime}$, and $\hat{\tau}$ replaced by $\tau$. From Lemma 3.5 we now get that (3.16) is valid with $l=k$ and $s=r_{k}^{\prime}$. Let $r_{k+1}=\frac{r_{k}}{c_{6} c_{7}}$. Then using (3.16) and the induction hypothesis we have

$$
\begin{aligned}
\left|\log \frac{u\left(x, \xi_{k+1}\right)}{u(x)}-\log \frac{u\left(y, \xi_{k+1}\right)}{u(y)}\right| & \leq\left|\log \frac{u\left(x, \xi_{k+1}\right)}{u\left(x, \xi_{k}\right)}-\log \frac{u\left(y, \xi_{k+1}\right)}{u\left(y, \xi_{k}\right)}\right| \\
+\left|\log \frac{u\left(x, \xi_{k}\right)}{u(x)}-\log \frac{u\left(y, \xi_{k}\right)}{u(y)}\right| & \leq k c_{5}\left(\frac{|x-y|}{r_{k+1}}\right)^{\alpha}
\end{aligned}
$$

whenever $x, y \in B\left(w, r_{k+1}\right) \cap G$. Thus by induction we get (3.21) with $k=m$. Since $u\left(\cdot, \xi_{m}\right)=v$ and $r_{m} \geq r / c$ by construction, we conclude that Theorem 1 is valid.

4. Applications of Theorem 1 to the Martin Boundary Problem. Letting $r \rightarrow \infty$ in Theorem 1 we get the following corollary.

Corollary 1. Let $p, \phi, G$ be as in Theorem $A$ and suppose that $u, v$ are positive $p$ harmonic functions in $G$ with continuous boundary values 0 on $\partial G$. Then $u=\lambda v$ for some $\lambda \in(0, \infty)$.

We note that Corollary 1 can be rephrased as stating that minimal positive $p$ harmonic functions relative to $\infty$ in a Lipschitz graph domain are unique. To 
be more specific we need some definitions. We say that $\Omega \subset \mathbf{R}^{n}$ is a bounded Lipschitz domain, provided there exists a finite set of balls $\left\{B\left(x_{i}, r_{i}\right)\right\}$, with $x_{i} \in \partial \Omega$ and $r_{i}>0$, such that $\left\{B\left(x_{i}, r_{i}\right)\right\}$ constitutes a covering of an open neighbourhood of $\partial \Omega$ and such that, for each $i$,

$$
\begin{aligned}
& \Omega \cap B\left(x_{i}, 4 r_{i}\right)=\left\{y=\left(y^{\prime}, y_{n}\right) \in \mathbf{R}^{n}: y_{n}>\phi_{i}\left(y^{\prime}\right)\right\} \cap B\left(x_{i}, 4 r_{i}\right), \\
& \partial \Omega \cap B\left(x_{i}, 4 r_{i}\right)=\left\{y=\left(y^{\prime}, y_{n}\right) \in \mathbf{R}^{n}: y_{n}=\phi_{i}\left(y^{\prime}\right)\right\} \cap B\left(x_{i}, 4 r_{i}\right),
\end{aligned}
$$

in an appropriate coordinate system and for a Lipschitz function $\phi_{i}$. The Lipschitz constant of $\Omega$ is defined to be $M=\max _{i}\left\|\left|\nabla \phi_{i}\right|\right\|_{\infty}$. $\tilde{u}$ is said to be a minimal positive $p$ harmonic function in the Lipschitz domain $\Omega$ relative to $w \in \partial \Omega$, provided $\tilde{u}>0$ is $p$ harmonic in $\Omega$ and $\tilde{u}$ has continuous boundary value 0 on $\partial \Omega \backslash\{w\} . \tilde{u}$ is said to be unique up to constant multiples if $\tilde{v}=\lambda \tilde{u}$ for some constant $\lambda$, whenever $\tilde{v}$ is a minimal positive $p$ harmonic function relative to $w \in \partial \Omega$. Finally we say that the Martin boundary of $\Omega$ can be identified with $\partial \Omega$, provided each $w \in \partial \Omega$ corresponds to a unique (up to constant multiples) minimal positive $p$ harmonic function. We note that for $p=2$ one can easily use Theorem A to get that the Martin boundary of a bounded Lipschitz domain $\Omega$ agrees with its topological boundary. Indeed if $w \in \partial \Omega$, and $u, v$ are minimal harmonic functions corresponding to $w$, one first uses Theorem A for harmonic functions to show that $\gamma=\inf _{\Omega} u / v>0$. Next one applies this result to $u-\gamma v, v$ in order to conclude that $u=\gamma v$. In the $p$ harmonic case though we need to use a variation of Theorem 1 with $B(w, r)$ replaced by $\Omega \backslash B(w, r)$. Moreover currently our proof is not strong enough to prove that the Martin boundary of a Lipschitz domain always agrees with its topological boundary when $p \neq 2$.

To state our results we need to introduce definitions. We call $\tilde{\Omega} \subset \Omega$ a nontangential approach region at $w \in \partial \Omega$ if the intersection of the closure of $\tilde{\Omega}$ and the closure of $\Omega$ equals $w$ and if, for some $\tilde{\eta}>0, d(x, \partial \Omega) \geq \tilde{\eta}|x-w|$ for all $x \in \tilde{\Omega}$. To indicate $w$ and $\tilde{\eta}$ we write $\tilde{\Omega}(w, \tilde{\eta})$. Using Theorem 1 and its proof, it is easily seen that if $u$ is a minimal positive $p$ harmonic function in $\Omega$ relative to $w \in \partial \Omega$ and $x \neq w \in \partial \Omega$, then there exists, $c, \delta, \delta^{\prime}$ and $\xi=\xi(x)$ with $|\xi|=1$, such that

$$
\begin{aligned}
& \text { (a) } \delta^{2} u(y) / d(y, \partial \Omega) \leq \delta|\nabla u(y)| \leq u(y) / d(y, \partial \Omega), \\
& \text { (b) } \delta^{\prime}|\nabla u(y)| \leq\langle\nabla u(y), \xi\rangle,
\end{aligned}
$$

when $y \in \Omega \cap B(x,|x-w| / c)$. Here $c, \delta, \delta^{\prime}$ depend only on $p, n, M$. From (4.1) one sees that there exists $\tilde{\eta}$ such that $u$ satisfies $(4.1)(a)$ in $\Omega \backslash \tilde{\Omega}(w, \tilde{\eta})$. Moreover, 
for each $y \in \Omega \backslash \tilde{\Omega}(w, \tilde{\eta})$ there is a $x \in \partial \Omega \backslash\{w\}$ with $y \in B(x,|x-w| / c)$ for which (4.1) (b) holds. Using this observation and a variation of the arguments in Theorem 1 we in [LN1] prove,

Theorem B. Let $\Omega \subset \mathbf{R}^{n}$ be a bounded Lipschitz domain with constant $M$ and let $u, \tilde{\Omega}(w, \tilde{\eta})$, be as above. Suppose there exist a sequence of positive numbers $\left\{\rho_{l}\right\}$ with $\lim _{l \rightarrow \infty} \rho_{l}=0$, a fixed number $\tilde{b}>1$ and $\delta>0$ such that (4.1) (a) holds for all $x \in \hat{\Omega}=\cup_{l} \tilde{\Omega}(w, \tilde{\eta}) \cap\left[B\left(w, \tilde{b} \rho_{l}\right) \backslash B\left(w, \rho_{l} / \tilde{b}\right)\right]$. If $\tilde{b}=\tilde{b}(p, n, M)$ is large enough, then $u$ is the unique minimal positive $p$ harmonic function relative to $w$ (up to constant multiples).

From Theorem B we see that the determination of the $p$ Martin boundary at a boundary point $w$ is reduced, for Lipschitz domains, to proving the existence of a certain sequence of positive numbers tending to zero and a corresponding minimal positive $p$ harmonic function satisfying the nondegeneracy condition (4.1) $(a)$ in $\hat{\Omega}$, for $\tilde{b}$ suitably large. We are able to verify this condition in a number of interesting cases. In particular in [LN1] we prove the following theorem.

Theorem C. Let $\Omega \subset \mathbf{R}^{n}$ be a bounded Lipschitz domain with Lipschitz constant $M$. Then the $p$ Martin boundary of $\Omega$ can be identified with the topological boundary of $\Omega$ in the following cases.

(1) $\Omega$ is convex,

(2) $\partial \Omega$ is $C^{1}$,

(3) $M=M(p)$ is small enough,

(4) $n=2$.

Also if $\partial \Omega$ has a tangent plane at $w$, then a minimal positive $p$ harmonic function relative to $w$ is unique up to constant multiples.

As for the proof of Theorem C we note that once (4.1) (a) is verified we can argue as in the proof of Theorem 1 to establish certain decay estimates for the oscillation of $u / v$ in $\Omega \backslash B\left(w, r^{\prime}\right), r^{\prime}$ small. Letting $r^{\prime} \rightarrow 0$ we then get $u=\lambda v$ for some real $\lambda$ (compare with Corollary 1 ). Finally we mention that in future papers we plan to establish the equivalence of the Martin and topological boundary in 
certain Reifenberg flat and Ahlfors regular NTA domains. Moreover, preliminary results show that this equivalence also holds for Cantor sets in $\mathbb{R}^{2}$ and certain ' smooth' $k$ dimensional sets $\subset \mathbb{R}^{n}$ when $n-p<k<n$ is a positive integer.

\section{Applications of Theorem 1 to Regularity - Free Boundary Problems.}

We need some more notation in order to state the results in [LN2]. Let $\Omega \subset \mathbf{R}^{n}$ be the Lipschitz domain in section 4 . If $w \in \partial \Omega, 0<r<r_{0}$ and $0<b<1$, we let

$$
\Gamma(w)=\Gamma_{b}(w)=\{y \in \Omega: d(y, \partial \Omega)>b|w-y|\} \cap B(w, 4 r) .
$$

Given a measurable function $k$ on $B(w, 4 r) \cap \Omega$ we define the nontangential maximal function $N(k): \partial \Omega \rightarrow \mathbf{R}$ for $k$ as

$$
N(k)(x)=\sup _{y \in \Gamma(x)}|k|(y) \text { whenever } x \in \Omega .
$$

Let $L^{q}(\partial \Omega \cap B(w, 4 r)), 1 \leq q \leq \infty$, be the space of functions which are integrable, with respect to Hausdorff $n-1$ measure $\left(H^{n-1}\right)$ on $B(w, 4 r) \cap \partial \Omega$, to the power $q$. Furthermore, given a measurable function $f$ on $B(w, 2 r) \cap \partial \Omega$, we say that $f$ is of bounded mean oscillation on $B(w, r) \cap \partial \Omega, f \in B M O(B(w, r) \cap \partial \Omega)$, if for all $x \in B(w, r) \cap \partial \Omega$ and $0<s \leq r$, there exists $0<A<\infty$ satisfying

$$
\int_{B(x, s) \cap \partial \Omega}\left|f-f_{B}\right| d H^{n-1} \leq A s^{n-1} .
$$

Here $f_{B}$ denotes the average of $f$ on $B(x, s) \cap \partial \Omega$ with respect to $H^{n-1}$ measure. The least such $A$ for which (5.2) holds will be denoted by $\|f\|_{B M O(B(w, r) \cap \partial \Omega)}$. Finally we say that $f$ is of vanishing mean oscillation on $B(w, r), f \in V M O(B(w, r) \cap$ $\partial \Omega$ ), provided for each $\epsilon>0$ there is a $\delta>0$ such that (5.2) holds with $A$ replaced by $\epsilon$ whenever $0<s<\min (\delta, r)$ and $x \in B(w, r)$.

In [LN2] we prove the following theorems.

Theorem D. Let $\Omega \subset \mathbf{R}^{n}$ be a bounded Lipschitz domain with constant $M$. Given $p, 1<p<\infty, w \in \partial \Omega, 0<r<r_{0}$, suppose that $u$ is a positive $p$ harmonic function in $\Omega \cap B(w, 4 r), u$ is continuous in $\bar{B}(w, 4 r) \cap \bar{\Omega}$ and $u=0$ on $B(w, 4 r) \cap \partial \Omega$. Then

$$
\lim _{y \in \Gamma(x), y \rightarrow x} \nabla u(y)=\nabla u(x)
$$


for $H^{n-1}$ almost every $x \in B(w, 4 r) \cap \partial \Omega$. Furthermore there exist $q>p$ and a constant $c, 1 \leq c<\infty$, which both only depend on $p, n$ and $M$ such that

(i) $N(|\nabla u|) \in L^{q}(B(w, 2 r) \cap \partial \Omega)$,

(ii) $\int_{B(w, 2 r) \cap \partial \Omega}|\nabla u|^{q} d H^{n-1} \leq c r^{(n-1)\left(\frac{p-1-q}{p-1}\right)}\left(\int_{B(w, 2 r) \cap \partial \Omega}|\nabla u|^{p-1} d H^{n-1}\right)^{q /(p-1)}$,

(iii) $\log |\nabla u| \in B M O(B(w, r) \cap \partial \Omega), \quad\|\log |\nabla u|\|_{B M O(B(w, r) \cap \partial \Omega)} \leq c$.

Theorem E. Let $\Omega \subset \mathbf{R}^{n}, M, p, w, r$ and $u$ be as in the statement of Theorem D. If, in addition, $\Omega$ is $C^{1}$ regular then

$$
\log |\nabla u| \in \operatorname{VMO}(B(w, r) \cap \partial \Omega) .
$$

Theorem F. Let $\Omega \subset \mathbf{R}^{n}, M, p, w, r$ and $u$ be as in the statement of Theorem $D$. Then there exists $M_{0}$, independent of $u$, such that if $M \leq M_{0}$ and if $\log |\nabla u| \in \operatorname{VMO}(B(w, r) \cap \partial \Omega)$, then the outer unit normal to $B(w, r) \cap \partial \Omega$ is in $\operatorname{VMO}(B(w, r / 2) \cap \partial \Omega)$.

We note that Theorem $\mathrm{D}$ and Theorem $\mathrm{E}$ are proved in [LN] for $p$ capacitary functions in starlike Lipschitz ring domains and in fact, given Theorem 1, modest modifications of the arguments of $[\mathrm{LN}]$ are needed to get Theorems D and E. Hence Theorem $\mathrm{F}$ is the main theorem in [LN2]. As for the proof of Theorem F, one uses Theorem 1, the assumption $\log |\nabla u| \in \mathrm{VMO}(B(w, r) \cap \partial \Omega)$ and blowup type arguments to show for arbitrary $w_{j} \in B(w, r / 2) \cap \partial \Omega$ and for a sequence of scales $\left\{r_{j}\right\}, r_{j} \rightarrow 0$, that $B\left(w_{j}, r_{j}\right) \cap \partial \Omega$ gets flater and flater, as $j$ tends to infinity, in a sense made precise in [LN2]. Finally we remark that we intend to generalize Theorems $D, E, F$, as in [KT, KT1, KT2] for $p=2$, to certain chord arc domains.

\section{REFERENCES}

[G] F. Gehring, $L^{p}$ integrability of the partial derivatives of a quasiconformal mapping, Acta Math. 130 (1973), 265-277.

[JK] D. Jerison and C. Kenig, Boundary behavior of harmonic functions in nontangentially accessible domains, Advances in Math. 46 (1982), 80-147. 
[KP] C. Kenig and J. Pipher, The Dirichlet problem for elliptic operators with drift term, Publ. Mat. 45 (2001), no. 1, 199-217.

[KT] C. Kenig and T. Toro, Harmonic measure on locally flat domains, Duke Math J. 87 (1997), 501-551.

[KT1] C. Kenig and T. Toro, Free boundary regularity for harmonic measure and Poisson kernels, Ann. of Math. 150 (1999), 369-454.

[KT2] C. Kenig and T. Toro, Poisson kernel characterization of Reifenberg flat chord arc domains, Ann. Sci. Ecole Norm. Sup. (4) 36 (2003), no. 3, 323 - 401.

[LN] J. Lewis and K. Nyström, Boundary Behaviour for p-Harmonic Functions in Lipschitz and Starlike Lipschitz Ring Domains, Annales Scientifiques de L'Ecole Normale Superieure, Volume 40, Issue 5, September-October 2007, 765-813.

[LN1] J. Lewis and K. Nyström, Boundary Behaviour and the Martin Boundary Problem for p-Harmonic Functions in Lipschitz domains, to appear in Annals of Mathematics.

[LN2] J. Lewis and K. Nyström, Regularity and Free Boundary Regularity for the p-Laplacian in Lipschitz and $C^{1}$-domains, Annales Acad. Sci. Fenn. Mathematica 33 (2008), 523 548.

John L. Lewis

Department of Mathematics

University of Kentucky

Lexington, KY 40506-0027, USA

E-mail: john@ms.uky.edu

Kaj Nyström

Department of Mathematics

Umeå University

S-90187 Umeå, Sweden

E-mail: kaj.nystrom@math.umu.se 